\title{
Intervista a Maria Cristina Mereu
}

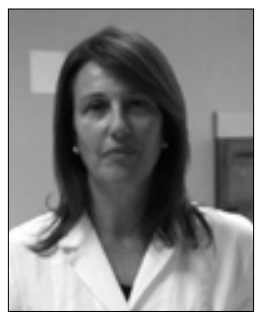

Maria Cristina Mereu, MD

P.O. NS Bonaria

San Gavino Monreale

mariacristina.mereu@asl6sanluri.it

Nel Centro ove presti attività qual è la percentuale di pazienti monitorati per CKD-MBD stadio 3-5 e quali marker biologici usi a tal fine?

Nel nostro Centro seguiamo circa 900 pazienti con CKD stadio 3-5. Nel 70\% dei pazienti valutiamo $\mathrm{Ca}, \mathrm{Pi}, \mathrm{FA} \mathrm{e}$ PTH. Ovviamente la frequenza del monitoraggio specie per PTH e FA avviene in base allo stadio di malattia e alla presenza d'alterazioni.

Nel Centro ove presti attività pratichi il dosaggio della $25(\mathrm{OH}) \mathrm{D}$ e/o della $1,25(\mathrm{OH})_{2} \mathrm{D} 3$ ?

Se sì, in quale tipo di paziente e in quale percentuale? Se no, perché ritieni di non doverla praticare?

Il dosaggio della $25(\mathrm{OH}) \mathrm{D}$ è praticato nel $4-5$ stadio a tutti, nei pazienti con età $>65$ anni e nei diabetici anche negli stadi più precoci di malattia $(2,3)$. La concentrazione serica di $25(\mathrm{OH}) \mathrm{D}$ costituisce il miglior indicatore dello stato vitaminico $D$ in quanto riflette i contributi sia cutanei sia alimentari. Il suo dosaggio pertanto assume un importante significato in quanto il livello serico $>30$ $\mathrm{ng} / \mathrm{mL}$ è in grado di evitare una stimolazione paratiroidea e di massimizzare l'assorbimento del calcio a livello intestinale.

Come recentemente dimostrato, il deficit vitaminico non riguarda solo gli anziani, ma è presente in diverse età sia nella popolazione generale che nei pazienti con CKD. Le raccomandazioni per la sua supplementazione, derivano dalle numerose e recenti osservazioni che confermano il ruolo essenziale della vit. D nel metabolismo minerale (prevenzione dell'IPS, riduzione del rischio di frattura), ma anche per i numerosi effetti extrascheletrici.

Infatti, i recettori per la vit. D (VDR) sono stati descritti in diversi organi: nei monociti-macrofagi, dove la vit. D sembra avere un ruolo antibatterico e immunomodulante; in alcune cellule epiteliali, dove regolerebbe la produzione di fattori apoptotici e antiaggreganti; a livello renale entra in circolo e può favorire una "down regulation" della sintesi di renina e/o stimolare la produzione di insulina da parte delle insule pancreatiche. Si presume inoltre, che la produzione "locale" di $1,25(\mathrm{OH})_{2} \mathrm{D} 3$, nella mammella, colon, prostata e altri tessuti, sia in grado di regolare un gran numero di geni che controllino la proliferazione, inibiscano la differenziazione e inducano l'apoptosi. Sono invece necessari studi randomizzati che confermino come una adeguata replezione vitaminica $\mathrm{D}$ possa ridurre la morbilità e mortalità cardiovascolare. sia nei pazienti con $\mathrm{CKD}$ che nella popolazione generale.

Il dosaggio dell' $1,25(\mathrm{OH})_{2} \mathrm{D} 3$, invece si è rivelato poco utile ai fini terapeutici nei nostri pazienti. Pertanto, questo dosaggio va riservato solo a casi particolari oltre che per la sua breve emivita (4-6 ore), anche perché influenzabile dall'uso di calcitriolo.

Applichi la correzione di 25(OH)D in caso di carenza? Perché, con quale protocollo e in quale tipo di paziente?

Il ripristino dei livelli di $25(\mathrm{OH}) \mathrm{D}$ dovrebbe essere il primo "step" nella terapia delle alterazioni del metabolismo minerale in tutti i pazienti dallo stadio 2 a 4 con lo scopo di prevenire l'iperparatiroidismo secondario (IPS), ma va effettuato anche negli stadi più avanzati di malattia e in dialisi per favorire i benefici extrascheletrici.

Le vitamine D2 (ergocalciferolo), la D3 (colecalciferolo) o il calcifediolo possono essere utilizzate per la supplementazione; i dosaggi variano in base al deficit e alla presenza di alcuni fattori di rischio (obesità, sindrome nefrosica, malassorbimento, uso di steroidi e anticonvulsivanti). Ad oggi però, non si conosce il dosaggio ideale che potrebbe favorire gli effetti benefici extrascheletrici. 
Nel Centro ove presti attività in quale percentuale o in quali casi clinici particolari pratichi la biopsia ossea?

Non facciamo la biopsia ossea ormai da diversi anni. Ma sono convinta che questo esame che costituisce il gold standard per la diagnosi delle alterazioni del turnover osseo, debba essere reintrodotto nei nostri Centri. Infatti, pur essendo una metodica invasiva, sarebbe decisamente utile per meglio comprendere le fratture da "fragilità", sempre più frequenti e a volte inspiegabili nei nostri pazienti, e quindi per consentire un approccio terapeutico adeguato. Inoltre l'esame fornirebbe maggiori chiarimenti sull'azione di alcuni farmaci di uso comune come i calciomimetici e/o chelanti a base di metalli (carbonato di lantanio), di cui ancora oggi non conosciamo gli effetti sull'osso nel lungo termine.

$\mathrm{Nel}$ Centro ove presti attività in quale percentuale $e$ in quali stadi di CKD usi indagini strumentali per la ricerca delle calcificazioni vascolari? Quali indagini utilizzi?

Nei pazienti incidenti e prevalenti in dialisi utilizziamo la valutazione dello score di calcificazione aortica (con il metodo di Kauppila), mediante radiografia laterale dell'addome lombare; e da circa un anno la eseguiamo anche nei pazienti in predialisi e nei diabetici. L'ecocardiogramma è un esame di routine non solo per la valutazione cardiaca, ma per il riscontro delle calcificazioni valvolari.

Nel Centro ove presti attività qual è la percentuale di pazienti in trattamento sostitutivo che resta nel range di normalità (secondo le linee guida SIN) per Ca, P e PTH?

Per il Calcio 60\%, Pi 65\%, PTH 45\%.

Nel Centro ove presti attività qual è la percentuale dei vari chelanti usati per ricercare il controllo del Ca-P nei pazienti in trattamento sostitutivo (anche in off-label treatment)?

Vengono utilizzati nel $45 \%$ sevelamer; nel 35\% calcio carbonato, e nel $10 \%$ in associazione.

Non vengono usati chelanti a base di gel di alluminio.

Nel Centro ove presti attività qual è la percentuale di pazienti in CKD 3-5 sottoposta a dieta ipoproteica per il controllo della iperfosforemia?

Una restrizione proteica variabile da 0,8 a $0,6 / \mathrm{gr} / \mathrm{kg} / \mathrm{die}$ di proteine con o senza prodotti aproteici, viene effettua- ta nel 50\% dei pazienti, con molta attenzione agli anzia$\mathrm{ni}$, che tendono già a limitare l'introito proteico spesso per problemi di masticazione e quindi possono andare incontro a malnutrizione.

Nel Centro ove presti attività quale percentuale di pazienti in trattamento sostitutivo che assumono vit-D o analoghi, calciomimetico, o entrambi?

Paracalcitolo 19\% , Calcitriolo 40\%, calcio mimetico 10\% sempre associato a vitamina $\mathrm{D}$.

Dopo oltre un trentennio di osservazioni, esperienze, studi e ricerche, la fisiopatologia dell'iperparatiroidismo secondario non è ancora del tutto chiarita. Calcio, fosforo, recettori della vit-D o recettori sensibili del calcio, ormoni fosfatotropi e forse l'esistenza di un recettore cellulare del fosforo, sono tutti "in carriera" assieme al primo attore il PTH (almeno nella cronologia storica): si è in grado di prevedere un ordine e chiarimento delle cose o dobbiamo ancora e solo predire?

Secondo l'ipotesi classica, durante la progressione della CKD, le alterazioni riguardanti i parametri del metabolismo minerale, come calcio e fosforo, e quelle ormonali come vit. D e PTH, erano in grado di determinare un quadro di IPS, che successivamente ha contribuito non solo allo sviluppo della malattia ossea, ma anche alle calcificazioni vascolari con aumento del rischio indipendente di morbilità e mortalità cardiovascolare. Poco chiara risultava la precoce riduzione della vit. D e alcuni stati di ipofosforemia precoce. Numerose e recenti acquisizioni hanno evidenziato l'esistenza di un meccanismo di regolazione ormonale aggiuntivo, costituito dall'FGF23 e il suo recettore Kloto, coinvolto nella fisiopatologia delle varie condizioni cliniche associate alla CKD. Pertanto oggi sappiamo che un'alterazione dell'omeostasi del fosforo costituisce cronologicamente il primum movens per l'instaurarsi dell'IPS. Infatti a ogni variazione di $\mathrm{Pi}$, segue un aumento precoce dell'FGF23 (già dal $2^{\circ}$ stadio,) che comporta una precoce riduzione della produzione di vit. $\mathrm{D}$ attiva, e conseguente mancato feed-back negativo, sia diretto, sulla sintesi e secrezione paratiroidea, che indiretto (attraverso la mancata attivazione dei CaSR ) sulla paratiroide. Tutti questi cambiamenti avvengono decisamente molto prima che si sviluppi iperfosfatemia, che invece è un evento molto tardivo (4-5 stadio). Del resto l'iperfosfatemia in questi ultimi dieci anni, è stato identificato come il maggior fattore di rischio di mortalità nei pazienti in dialisi in quanto, oltre 
ad essere il principale determinante dell'IPS e della malattia dell'osso, direttamente promuove le calcificazioni cardiovascolari. La presenza di calcificazioni vascolari è stata confermata in tutte le fasi dell'CKD. Recenti studi epidemiologici e osservazionali hanno dimostrato un aumento del rischio di mortalità per valori di Pi del tutto "normali"; inoltre è stata evidenziata una correlazione indipendente tra aumento del rischio di mortalità e severità di malattie cardiovascolari per ogni variazione di $1 \mathrm{mg} / \mathrm{dL}$ di Pi (entro il range di normalità), non solo nei soggetti con $\mathrm{CKD}$, ma anche nella popolazione normale. E evidente quindi l'esistenza di un link tra fosforo e calcificazione, non solo per elevati livelli serici di fosfato, ma anche in presenza di tutte quelle modificazioni di alterata omeostasi di questo ione già nelle fasi più precoci di malattia. Alla luce di ciò, sembra plausibile pensare che molto presto sarà indispensabile una ulteriore revisione e riduzione dei valori "normali" di riferimento del $\mathrm{Pi}$, pur in mancanza di studi prospettici randomizzati che confermino i reali vantaggi sugli outcome clinici.

Pertanto l'attenzione nel prossimo futuro sarà rivolta sempre più al chiarimento di tutti i meccanismi regolatori dell'asse intestino, osso, rene, paratiroide fin dalle fasi iniziali della CKD e alla miglior individuazione di altri fattori fosfaturici (di origine intestinale).

Infine sono necessari studi randomizzati per valutare l'efficacia di un precoce intervento terapeutico mirato a ristabilire l'omeostasi del Pi e il blocco della cascata di eventi negativi derivati dalla sua alterazione.

FGF23. Ł̀ stato detto che nell'uremico avrebbe le carte in regola per diventare un indicatore di esposizione al fosforo... come l'Hb glicata nel diabetico. Cosa ci dobbiamo/possiamo aspettare dall'entrata nell'uso clinico di tale marker?

Come ho già detto la scoperta dell'FGF23 in pochi anni ha drasticamente cambiato la conoscenza dei disordini del metabolismo minerale.

Il suo precoce e graduale incremento, costituisce un meccanismo di inappropriata compensazione corporea per mantenere l'omeostasi del Pi ogni qualvolta questa sia alterata nel corso della CKD. Come il PTH, è un ormone fosfaturico, ma la riduzione precoce della vit. D costituisce la più importante conseguenza del suo aumento. Restano a oggi comunque irrisolte una serie di questioni e tra queste: chi è il principale regolatore dell'FGF23? Esiste un sensore per il fosforo che determina il suo aumento? Quali sono i livelli normali in rapporto a età nei pazienti normali e nelle varie fasi della CKD? In che modo l'FGF23 inibisce il PTH? La sua azione inibitoria si esplica anche nei confronti della 1alpha-idrossilasi periferica?

In conclusione, sia perché non conosciamo il metodo di dosaggio ideale, sia per gli elevati costi che questo comporta, ritengo che l'utilizzo della fosfaturia sia una metodica semplice, di facile utilizzo e in grado di fornire nella pratica clinica una precisa informazione riguardo il carico di fosfati con la dieta.

Calciomimetici. Potenti, affidabili, indispensabili: quando come e perché?

Il calciomimetico ha rappresentato una delle più importanti opportunità terapeutiche nella terapia dell'IPS degli ultimi anni. Infatti modulando in maniera allosterica il CaSR, si è dimostrato efficace nel ridurre e mantenere nel lungo termine $\mathrm{PTH}, \mathrm{Ca}, \mathrm{P}$, secondo i livelli raccomandati dalle K/DOQI. Inoltre, se tollerato, il suo utilizzo, in associazione con calcitriolo e/o paracalcitolo, consente non solo una miglior gestione del $\mathrm{Ca}$ e $\mathrm{P}$, ma permette di ridurre le dosi della vit. $\mathrm{D}$, con somministrazioni più fisiologiche e più continuative nel tempo, che garantiscono un maggior successo terapeutico e minor ricorso alla terapia chirurgicia (PTX). Resta ancora da definire l'azione del calciomimetico sulle calcificazioni e quindi il suo impatto sul sistema cardiovascolare, sul turnover osseo, e le sue possibili interferenze sui CaSR presenti sugli altri sistemi cellulari.

Nonostante tutto (linee guida comprese, SIN, K/DOKI, KDIGO) nella maggioranza dei casi $i$ target terapeutici per calcio, fosforo e PTH non vengono centrati e soprattutto mantenuti. Quale commento ti stimola tale insufficiente raggiungimento?

Negli ultimi anni abbiamo sicuramente migliorato il raggiungimento dei target per calcio, e fosforo e PTH grazie alla introduzione di nuove strategie terapeutiche. D'altronde la complessa definizione di CKDMBD richiede un approccio terapeutico molteplice di cui ancora oggi non siamo pienamente soddisfatti. Infatti se da un lato siamo riusciti a curare meglio l'iperparatiroidismo, non sappiamo ancora quanto le terapie attuali siano altrettanto efficaci sugli altri aspetti della CKD-MBD: l'impatto sull'osso, sulle calcificazioni cardiache e vascolari, e sulla mortalità. 


\section{Commento conclusivo}

Negli ultimi anni la ricerca sul metabolismo minerale nella CKD ha chiarito molti punti oscuri, ma ha creato nuovi dubbi sull'approccio terapeutico.

Sembra comunque ormai chiaro che l'omeostasi degli ioni è strettamente regolata da un sistema perfetto ma piuttosto complicato e ancora non completamente chiarito tra ossa, reni, intestino e paratiroidi, che coinvolge una serie di trasportatori specifici, recettori, citochine e ormoni. Questo sofisticato meccanismo consente di stabilire un legame tra le alterazioni dell'omeostasi degli ioni e il processo di calcificazione vascolare, che si verifica fin dalle fasi più precoci della $\mathrm{CKD}$ e allo stesso modo, colpisce la popolazione generale. Il crescente interesse per i meccanismi patogenetici dell'IPS nelle fasi iniziali dell'insufficienza renale è evidente, dal momento che le strategie preventive o terapeutiche per questo "complesso" squilibrio metabolico potrebbero migliorare non solo le lesioni ossee, ma anche l'outcome clinico, specie quello cardiovascolare, di molti pazienti con CKD e, eventualmente, anche dei soggetti "sani”. 